\title{
Biomimetic Mineralization on a Macroporous Cellulose-Based Matrix for Bone Regeneration
}

\author{
Odeta Petrauskaite, ${ }^{1}$ Pedro de Sousa Gomes, ${ }^{2}$ Maria Helena Fernandes, ${ }^{2}$ \\ Gintaras Juodzbalys, ${ }^{3}$ Arturas Stumbras, ${ }^{3}$ Julius Maminskas, ${ }^{3}$ \\ Jolanta Liesiene, ${ }^{1}$ and Marco Cicciù ${ }^{4}$ \\ ${ }^{1}$ Department of Organic Technology, Kaunas University of Technology, Radvilenu pl. 19, 50254 Kaunas, Lithuania \\ ${ }^{2}$ Laboratory for Bone Metabolism and Regeneration, Faculty of Dental Medicine, University of Porto, Rua Dr. Manuel Pereira da Silva, \\ 4200-392 Porto, Portugal \\ ${ }^{3}$ Department of Oral and Maxillofacial Surgery, Lithuanian University of Health Sciences, Eiveniu str. 2, 50009 Kaunas, Lithuania \\ ${ }^{4}$ Human Pathology Department, Dental School, University of Messina, Messina IT, Policlinico G. Martino, Via Consolare Valeria, \\ 98100 Messina, Italy
}

Correspondence should be addressed to Marco Cicciù; acromarco@yahoo.it

Received 10 June 2013; Revised 15 August 2013; Accepted 21 August 2013

Academic Editor: Esmaiel Jabbari

Copyright (C) 2013 Odeta Petrauskaite et al. This is an open access article distributed under the Creative Commons Attribution License, which permits unrestricted use, distribution, and reproduction in any medium, provided the original work is properly cited.

\begin{abstract}
The aim of this study is to investigate the biomimetic mineralization on a cellulose-based porous matrix with an improved biological profile. The cellulose matrix was precalcified using three methods: (i) cellulose samples were treated with a solution of calcium chloride and diammonium hydrogen phosphate; (ii) the carboxymethylated cellulose matrix was stored in a saturated calcium hydroxide solution; (iii) the cellulose matrix was mixed with a calcium silicate solution in order to introduce silanol groups and to combine them with calcium ions. All the methods resulted in a mineralization of the cellulose surfaces after immersion in a simulated body fluid solution. Over a period of 14 days, the matrix was completely covered with hydroxyapatite crystals. Hydroxyapatite formation depended on functional groups on the matrix surface as well as on the precalcification method. The largest hydroxyapatite crystals were obtained on the carboxymethylated cellulose matrix treated with calcium hydroxide solution. The porous cellulose matrix was not cytotoxic, allowing the adhesion and proliferation of human osteoblastic cells. Comparatively, improved cell adhesion and growth rate were achieved on the mineralized cellulose matrices.
\end{abstract}

\section{Introduction}

Several bone grafts techniques are currently available for favouring regenerative processes on bone trauma, or for promoting healing between two bones across a diseased joint, and also for having new bone formation on site affected by disease, infection, or resection. Nowadays, great attention is focused on polymer/ceramic three-dimensional scaffolds for bone tissue regeneration [1-7]. It is well known that by choosing an appropriate polymer [8] and ceramic, for example, hydroxyapatite (HA) due to its excellent osteoconductivity, biocompatibility, and bioactivity [9], it is possible to fabricate well functionalizing scaffolds. This composite material must be nontoxic, compatible with the surrounding biological systems, and biodegradable. The scaffold has to be a 3D interconnected porous structure capable of promoting cell adhesion, proliferation and vascularization, and enabling a controlled supply of bioactive substances, which may influence the behaviour of incorporated or ingrown cells $[10,11]$.

The cellulose matrix shares a number of these advantages. It is an abundant, renewable, biocompatible, nontoxic, and biodegradable polymer [12]. Moreover, it has good mechanical properties because of the strong hydrogen bonding between the cellulose chains [13]. However, it has no bioactivity within the bone tissue [14]. Several studies have carried out research to establish a direct bond between developed material and natural bone tissue: this consists 
of the development of a hydroxyapatite layer by means of biomimetic mineralization $[9,11,15]$. As such, a simulated electrolyte body fluid solution (SBF) with ion concentrations similar to those of human blood plasma is used. An increased concentration of calcium ions is also required as these accelerate the nucleation rate of the hydroxyapatite crystals.

To date, covering scaffold surfaces with hydroxyapatite layer by the biomimetic route for the bone-bonding ability continues to be of great interest. Polymers with hydrophilic polar (e.g., hydroxyl, carboxyl, and silanol) groups are used due to their capacity to induce apatite nucleation [16-19]. Hong et al. [19] have revealed that bacterial cellulose (BC) and hydroxyapatite composites could be prepared by soaking $\mathrm{BC}$ in $\mathrm{CaCl}_{2}$ solution prior to the biomimetic mineralization. However, introduced carboxyl functional groups on BC by TEMPO ((2,2,6,6-tetramethylpiperidine-1-oxyl)mediated oxidation) and combined with calcium ions could enhance the rate of apatite nucleation as described by Nge and Sugiyama [20]. Greater numbers of smaller sized crystals grew on BC-TEMPO-Ca than did on native BC. Leonor et al. [21] successfully prepared bioactive chitosan microparticles with bone-bonding properties by introducing silanol groups combined with calcium ions, which were then soaked in a simulated body fluid. An apatite layer formed on their surfaces just under one day.

Consistent bone regeneration for the treatment of combined vertical and horizontal defects without the application of large bone grafts, exogenous growth factors, or cells still remains a challenge for clinicians and surgeons.

Several bone substitutes are currently available like carriers or scaffold. However, the effectiveness osteoconductivity property of the bone graft is still related with the microstructure of each material, and autogenous bone still remains the "gold standard." The study about bone substitutes as a valid alternative to autogenous bone grafts is connected to several clinical challenges. Clinicians and surgeons would eliminate the need to harvest bone from body sites when performing oral and maxillofacial regenerative surgery and the patient's pain and discomfort associated to these procedures $[3,4,6$, $16]$.

In this study, a macroporous cellulose matrix with an improved biological profile and performed biomimetic mineralization to induce hydroxyapatite formation was prepared. Precalcification of the matrix was done by means of three different methods: (i) treating the samples with $\mathrm{CaCl}_{2}$ and $\left(\mathrm{NH}_{4}\right)_{2} \mathrm{HPO}_{4}$ solutions; (ii) storing the carboxymethylated cellulose matrix in a saturated calcium hydroxide solution; (iii) treating the matrix with a calcium silicate solution. In addition, the biological performance of the porous cellulose matrix and the developed mineralized cellulose subtracts was evaluated with human osteoblastic cells regarding cell adhesion, morphology, and proliferation. Final aim of the paper is to investigate how this cellulose matrix could be predictable used for future application on large bone defects. The obtaining of a valid carrier or scaffold may be helpful for clinicians avoiding autogenous bone graft for other body sites reducing patient's discomfort, cost, and morbidity.

\section{Materials and Methods}

2.1. Materials. For this study, cellulose diacetate (DAC, $55 \%$ bond acetic acid) was obtained from Roshal (Russia). MG63 cells were obtained from the American Type Culture Collection (ATCC CRL-1427). Ammonia water $\left(\mathrm{NH}_{3} \cdot \mathrm{H}_{2} \mathrm{O}, 25 \%\right)$ and acetone $\left(\mathrm{CH}_{3} \mathrm{COCH}_{3}\right)$ were purchased from Stanlab (Poland). Ethanol $\left(\mathrm{C}_{2} \mathrm{H}_{5} \mathrm{OH}, 96.3 \%\right)$ was purchased from Stumbras (Lithuania). All other reagents were purchased from the Sigma-Aldrich Company.

2.2. Preparation of a Porous Cellulose Matrix. Cellulosebased gel was prepared by the regeneration of cellulose from cellulose diacetate (DAC) according to the patent [22]. For this purpose, $25 \mathrm{~g}$ of DAC was dissolved in $265 \mathrm{~mL}$ of an acetone-ammonia water mixture and conserved until a solid gel was formed. The gel was thoroughly rinsed with water. In order to create the highly porous matrix, cylindrical gel samples, with a diameter of about $12 \mathrm{~mm}$ and a thickness of $6 \mathrm{~mm}$, were washed with the ethanol-water solution $(1: 4 \mathrm{v} / \mathrm{v})$ and lyophilized in the Christ ALPHA 2-4 LSC freeze dryer.

2.3. Porosity. The porosity of the cellulose matrix was determined by the liquid displacement method [23]. Ethanol was used as a displacement liquid because it penetrates easily into the pores and does not cause shrinking or swelling of the matrix. The dehydrated sample (approx. $0.1 \mathrm{~g}$ ) was placed in a graduated cylinder containing $4 \mathrm{~mL}\left(V_{1}\right)$ of ethanol and stored at $37^{\circ} \mathrm{C}$ for $6 \mathrm{hrs}$. Before weighting, the tightly sealed cylinder containing the immersed sample was placed into an ultrasonic bath for 10 minutes to force ethanol into the pores of the polymer matrix. The total volume was then recorded as $V_{2}$. Subsequently, the sample was taken out, and the volume of ethanol was recorded as $V_{3}$. The porosity $(P)$ of the cellulose matrix was calculated using the following:

$$
P(\%)=\frac{V_{1}-V_{3}}{V_{2}-V_{3}} \times 100 .
$$

2.4. Water Retention. Water retention of the prepared matrix was determined using phosphate buffer solution ( $\mathrm{pH} 7.4)$ in order to simulate physiological conditions. The dehydrated sample (approx. 0.1g $\left(W_{1}\right)$ ) was immersed in a phosphate buffer solution at $37^{\circ} \mathrm{C}$ for $24 \mathrm{hrs}$. Before weighting, the bottle containing the sample was placed into an ultrasonic bath for 10 minutes to ensure that every pore was filled with the solution. Lastly, the sample was wiped with filter paper and weighted as $W_{2}$. Water retention (WR) was then defined by:

$$
\mathrm{WR}(\%)=\frac{W_{2}-W_{1}}{W_{1}} \times 100 .
$$

2.5. Incubation of Cellulose in $\mathrm{CaCl}_{2}$ and $\left(\mathrm{NH}_{4}\right)_{2} \mathrm{HPO}_{4}$ Solutions. The precalcification and formation of an amorphous calcium phosphate layer, for further conversion to hydroxyapatite in a simulated body fluid, were carried out by sequentially immersing the dehydrated cellulose samples (approx. $0.5 \mathrm{~g}$ ) in $50 \mathrm{~mL}$ of $0.2 \mathrm{~mol} \mathrm{~L}^{-1} \mathrm{CaCl}_{2}$ solution and $50 \mathrm{~mL}$ of 
$0.2 \mathrm{~mol} \mathrm{~L}^{-1}\left(\mathrm{NH}_{4}\right)_{2} \mathrm{HPO}_{4}$ solution at room temperature for $1.5 \mathrm{hrs}$ per step. After soaking, the samples were rinsed with distilled water and dried at $105^{\circ} \mathrm{C}$ for $24 \mathrm{hrs}$.

\subsection{Carboxymethylation of the Cellulose Matrix and Treat-} ment with a Saturated Calcium Hydroxide Solution. The cellulose matrix was carboxymethylated with chloroacetic acid in the presence of sodium hydroxide. For this purpose, $1 \mathrm{~g}$ of dehydrated cellulose was immersed in a mixture of $2.75 \mathrm{~mL}$ of $40 \% \mathrm{NaOH}$ and $30 \mathrm{~mL}$ of ethanol. The mixture was heated to $50^{\circ} \mathrm{C}$, and $1.29 \mathrm{~g}$ of chloroacetic acid was added. Carboxymethylation was performed at $60^{\circ} \mathrm{C}$ for $2.5 \mathrm{hrs}$. The reaction product was washed with distilled water until neutral $\mathrm{pH}$ was obtained and then lyophilized. The amount of carboxymethyl groups was determined using the Back Titration Method as detailed in the literature [24].

Furthermore, the activation of the carboxymethylated matrix was performed by immersing samples (approx. $0.5 \mathrm{~g}$ ) into $50 \mathrm{~mL}$ of a saturated $\mathrm{Ca}(\mathrm{OH})_{2}$ solution at $0^{\circ} \mathrm{C}$ for $72 \mathrm{hrs}$ [25]. After incubation, samples were rinsed with distilled water and dried at $105^{\circ} \mathrm{C}$ for $24 \mathrm{hrs}$.

2.7. Cellulose Treatment with Calcium Silicate Solution. The silanol groups were incorporated into the cellulose matrix by soaking approximately $0.5 \mathrm{~g}$ of the cellulose matrix in $50 \mathrm{~mL}$ of the calcium silicate solution [26] with ratio $\mathrm{Si}\left(\mathrm{OC}_{2} \mathrm{H}_{5}\right)_{4}: \mathrm{H}_{2} \mathrm{O}: \mathrm{C}_{2} \mathrm{H}_{5} \mathrm{OH}: \mathrm{HCl}: \mathrm{CaCl}_{2}$ 1:4: $4: 0.014: 0.2$ $\mathrm{w} / \mathrm{w}$ for $3 \mathrm{hrs}$ at $37^{\circ} \mathrm{C}$. Polymer modification was followed by tetraethoxysilane hydrolysis and condensation reactions. After separation from the solution, the samples were dried at $105^{\circ} \mathrm{C}$ to a constant weight.

2.8. Biomimetic Mineralization. In vitro biomimetic mineralization was carried out by immersing the samples into a simulated body fluid (SBF) with a concentration factor of $1.5(1.5 \mathrm{x}$ $\mathrm{SBF})$ and the following ion concentrations $\left[10^{-3} \mathrm{~mol} \mathrm{~L}^{-1}\right]$ : $\mathrm{Na}^{+}=213.0 ; \mathrm{K}^{+}=7.5 ; \mathrm{Ca}^{2+}=3.75 ; \mathrm{Mg}^{2+}=2.25 ; \mathrm{Cl}^{-}=221.7$; $\mathrm{HPO}_{4}{ }^{2-}=1.5 ; \mathrm{HCO}_{3}{ }^{-}=6.3 ; \mathrm{SO}_{4}{ }^{2-}=0.75$. The $1.5 \mathrm{x}$ SBF solution was prepared by dissolving the calculated amounts of $\mathrm{NaCl}, \mathrm{KCl}, \mathrm{CaCl}_{2}, \mathrm{MgCl}_{2} \cdot 6 \mathrm{H}_{2} \mathrm{O}, \mathrm{NaHCO}_{3}, \mathrm{~K}_{2} \mathrm{HPO}_{4} \cdot 3 \mathrm{H}_{2} \mathrm{O}$, $\mathrm{Na}_{2} \mathrm{SO}_{4}$ in distilled water. The solution was buffered with Tris/ $\mathrm{HCl}$ to $\mathrm{pH} 7.4$ at $37^{\circ} \mathrm{C}$. The samples were incubated in $1.5 \mathrm{x} \mathrm{SBF}$ at $37^{\circ} \mathrm{C}$ for up to 14 days. The electrolyte solution was refreshed once a week. After separation from the solution, the samples were rinsed with distilled water and dried. The mass increase (MI) was calculated using:

$$
\operatorname{MI}(\%)=\frac{m_{1}-m_{0}}{m_{0}} \times 100,
$$

where $m_{0}$ and $m_{1}$ are the masses of the samples before and after the mineralization process, respectively.

2.9. Characterization. Infrared spectroscopy (IR) was used to analyse the chemical structure of cellulose before and after modification. Four mg of cellulose was mixed with $200 \mathrm{mg}$ of $\mathrm{KBr}$ for the preparation of transparent pellets. All spectra were recorded in the range from 4000 to $400 \mathrm{~cm}^{-1}$ on a Perkin Elmer FT-IR spectrometer.
X-ray diffraction (XRD) was used for identification of the calcium phosphate phase. Diffraction patterns were recorded on a DRON-6 using a $\mathrm{Cu} \mathrm{K}_{\alpha}$ radiation at $30 \mathrm{kV}$ and $20 \mathrm{~mA}$.

A high-resolution field emission scanning electron microscope FEI Quanta 200 FEG with a Schottky type electron gun was used to observe the morphology of polymer and to detect hydroxyapatite.

2.10. In Vitro Biological Evaluation. In vitro biological evaluation was performed using MG63 osteoblastic cells. Cells were cultured until the adequate confluence (around 80\%), in the $\alpha$-MEM culture medium containing $10 \%$ fetal bovine serum, $100 \mu \mathrm{g} / \mathrm{mL}$ penicillin, $10 \mathrm{IU} / \mathrm{mL}$ streptomycin, $2.5 \mu \mathrm{g} / \mathrm{mL}$ fungizone, and $50 \mu \mathrm{g} / \mathrm{mL}$ ascorbic acid, at $37^{\circ} \mathrm{C}$, in a humidified atmosphere of $5 \% \mathrm{CO}_{2}$ in air. For a subculture, the cell monolayer was washed twice with a phosphate-buffered saline (PBS) and incubated with a trypsin-EDTA solution (0.05\% trypsin, $0.25 \%$ EDTA) for 5 minutes at $37^{\circ} \mathrm{C}$ to ensure cell detachment. Cells were followed counting by a hemocytometer and resuspended in the culture medium. The cell suspension was used in the biological evaluation of the cellulose porous scaffolds.

The scaffolds-the cellulose matrix (control) and biomimetic HA-covered cellulose-were placed on the bottom of the wells of 24-well culture plates (one scaffold/well) and were seeded with MG63 osteoblastic cells $\left(10^{5}\right.$ cells $\left.\mathrm{cm}^{2}\right)$. Seeded scaffolds were cultured for 7 days in the experimental conditions described above. Colonized materials were evaluated throughout the culture time by confocal laser scanning microscopy (CLSM), scanning electron microscopy (SEM), and DNA content, in order to address the cell morphology, the F-actin cytoskeleton organization, and the cell proliferation.

CLSM and SEM Observation. For CLSM assessment, colonized scaffolds were fixed (3.7\% paraformaldehyde, $15 \mathrm{~min}$ ). Cell cytoskeleton filamentous actin (F-actin) was visualized by treating the cells with Alexa Fluor 488 Phalloidin (1:20 dilution in PBS, $1 \mathrm{~h}$ ) and counterstaining with propidium iodide $\left(1 \mu \mathrm{g} \mathrm{mL}{ }^{-1}, 10 \mathrm{~min}\right)$ for cell nuclei labelling. Labelled cultures were mounted in Vectashield and examined with a Leica SP2 AOBS (Leica Microsystems) microscopy. For SEM observation, samples were fixed (1.5\% glutaraldehyde in $0.14 \mathrm{M}$ sodium cacodylate buffer, $\mathrm{pH}=7.3,10 \mathrm{~min}$ ), dehydrated in graded alcohols, critical-point dried, sputtercoated with an Au/Pd thin film (SPI Module Sputter Coater equipment), and observed in a high resolution (Schottky) Environmental Scanning Electron Microscope (Quanta 400 FEG ESEM).

DNA Content. DNA was analyzed by the PicoGreen DNA quantification assay (Quant-iT PicoGreen dsDNA Assay Kit, Molecular Probes Inc., Eugene), according to manufacturer's instructions. Cultures were treated with Triton X-100 (0.1\%) (Sigma), and fluorescence was measured on an Elisa reader (Synergy HT, Biotek) at wavelengths of 480 and $520 \mathrm{~nm}$, excitation and emission, respectively, and corrected for fluorescence of reagent blanks. The amount of DNA was 


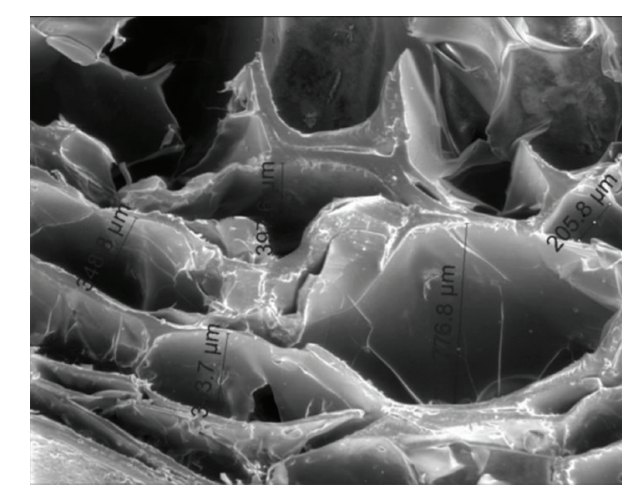

HV 20.00 kW-mag 100x-spot 5.0-det LFD-Quanta 200 FEG

FIGURE 1: SEM image of the macroporous cellulose used in the study.

calculated by extrapolating a standard curve obtained by running the assay with the given DNA standard.

Statistical Analysis. Three independent experiments were performed; in each experiment, three replicas were accomplished for the DNA quantification assay and two replicas for the qualitative assays (CLSM and SEM). DNA content is presented as mean \pm standard deviation (SD). Groups of data were evaluated using a two-way analysis of variance (ANOVA), and no significant differences in the pattern of the cell behavior were found. Statistical differences between experimental groups were assessed by Bonferroni's method. Values of $P \leq 0.05$ were considered statistically significant.

\section{Results and Discussion}

3.1. Characterization of the Cellulose Matrix. A cellulosebased gel was lyophilized in selected conditions, and the porous matrix was created with a porosity of $80 \%$ ensuring space for vascularization and bone ingrowth.

The scanning electron microscopy (SEM) micrograph revealed that the prepared matrix was composed of different pore sizes, ranging up to $780 \mu \mathrm{m}$ (Figure 1). Furthermore, the results of water retention showed that the prepared matrix could absorb more than $5 \mathrm{~g}$ of water per $1 \mathrm{~g}$ of the absolutely dried sample. This property substantiates high hydrophilicity of the matrix which reveals biocompatibility with biological systems.

3.2. Biomimetic Mineralization on the Cellulose Matrix. Mineral phase acts as a bonding layer to the bone; thus, it is very important to create such layer on cellulose scaffolds since cellulose itself does not have this ability.

In order to induce the mineralization process, the matrix was precalcified using three different methods: (i) treating freeze-dried cellulose samples with $\mathrm{CaCl}_{2}$ and $\left(\mathrm{NH}_{4}\right)_{2} \mathrm{HPO}_{4}$ solutions; (ii) storing the carboxymethylated cellulose matrix in a saturated $\mathrm{Ca}(\mathrm{OH})_{2}$ solution; (iii) treating the lyophilized samples with a calcium silicate solution in order to introduce silanol groups and combine them with calcium ions.

Figure 2 shows IR spectra of the cellulose matrix samples used for biomimetic mineralization. The broad absorption

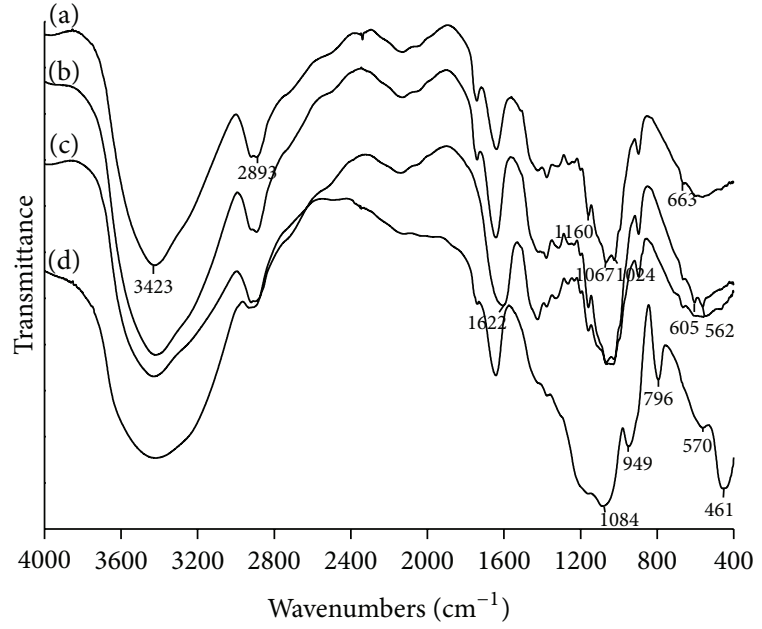

FIGURE 2: The spectra of (a) cellulose; (b) cellulose precalcified with $\mathrm{CaCl}_{2}$ and $\left(\mathrm{NH}_{4}\right)_{2} \mathrm{HPO}_{4}$ solutions; (c) carboxymethylated cellulose precalcified with a saturated $\mathrm{Ca}(\mathrm{OH})_{2}$ solution; (d) cellulose precalcificated with the calcium silicate solution.

band in the $3500-3200 \mathrm{~cm}^{-1}$ range was assigned to the stretching vibration of the $\mathrm{OH}$ group of cellulose. The band, at $2893 \mathrm{~cm}^{-1}$, corresponds to the symmetric stretching vibration of $\mathrm{CH}_{2}$ groups. The absorption band due to the asymmetric $\mathrm{C}-\mathrm{O}-\mathrm{C}$ stretching vibration appears at $1160 \mathrm{~cm}^{-1}$. The stretching of C-O appears at $1024 \mathrm{~cm}^{-1}$ and $1067 \mathrm{~cm}^{-1}$.

After treating the cellulose matrix with $\mathrm{CaCl}_{2}$ and $\left(\mathrm{NH}_{4}\right)_{2} \mathrm{HPO}_{4}$ solutions, new absorption peaks at $602 \mathrm{~cm}^{-1}$ and $562 \mathrm{~cm}^{-1}$ appeared in the spectra, confirming the formation of calcium phosphate. The spectrum of carboxymethylated cellulose (carboxymethyl groups amount $1.5 \mathrm{mmol} / \mathrm{g}$ ) activated with $\mathrm{Ca}(\mathrm{OH})_{2}$ displayed an absorption band at $1622 \mathrm{~cm}^{-1}$, which represented the symmetric stretching vibration of a carboxyl group. Figure 2(d) shows the IR spectra of cellulose after its treatment with the calcium silicate solution. Absorption bands at $1084 \mathrm{~cm}^{-1}$ and $796 \mathrm{~cm}^{-1}$ can be seen, which correspond to the asymmetric and symmetric stretching vibrations of $\mathrm{Si}-\mathrm{O}-\mathrm{Si}$, respectively. The band at $461 \mathrm{~cm}^{-1}$ is associated with $\mathrm{Si}-\mathrm{O}-\mathrm{Si}$ bending vibrations, while an absorption band at $949 \mathrm{~cm}^{-1}$ corresponds to the $\mathrm{Si}-\mathrm{OH}$ stretching vibration. This is the result of tetraethoxysilane hydrolysis and condensation.

The cellulose matrix samples were immersed in a simulated body fluid with a concentration factor of 1.5 (1.5x SBF) for up to 14 days. IR spectra of all cellulose samples after 14 days of mineralization in $1.5 \mathrm{x}$ SBF showed an absorption band in the $570-602 \mathrm{~cm}^{-1}$ range, which could be attributed to $\mathrm{PO}_{4}{ }^{3-}$ group (Figure 3). Apatite also gives absorption band in the $1000-1100 \mathrm{~cm}^{-1}$ region due to $\mathrm{PO}_{4}{ }^{3-}$ group. However, it should be noted that cellulose has intensive peaks in this region, which overlap with the expected peaks.

Following on, the mineralized cellulose samples were examined by an X-ray diffraction. The typical diffraction peaks of cellulose appeared at $12.4^{\circ}, 20.7^{\circ}, 21.1^{\circ}$, and $34.9^{\circ}$ in $2 \theta$ 
TABLE 1: Mass increase on the cellulose matrix after the mineralization process.

\begin{tabular}{lccc}
\hline Time, days & $\begin{array}{c}\text { Matrix pretreated with the } \\
\text { calcium silicate solution }\end{array}$ & $\begin{array}{c}\text { Mass increase, } \% \\
\text { and phosphate ions solutions }\end{array}$ & $\begin{array}{c}\text { Carboxymethylated matrix pretreated } \\
\text { with the calcium hydroxide solution }\end{array}$ \\
\hline 2 & 2 & 1 & 4 \\
7 & 3 & 3 & 7 \\
14 & 6 & 6 & 12 \\
\hline
\end{tabular}

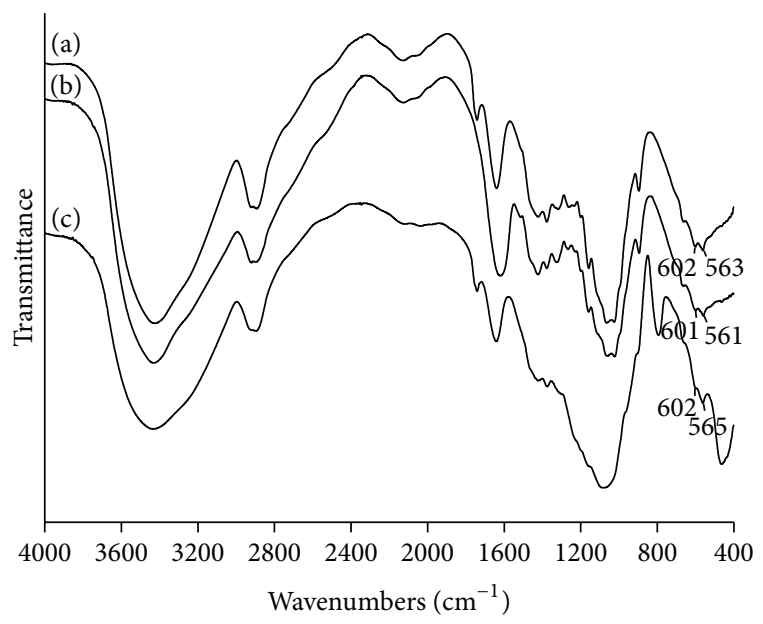

FIGURE 3: The IR spectra of (a) mineralized cellulose pretreated with calcium and phosphate ions solutions; (b) mineralized carboxymethylated cellulose pretreated with a saturated $\mathrm{Ca}(\mathrm{OH})_{2}$ solution; (c) mineralized cellulose pretreated with the calcium silicate solution.

(Figure 4(a)) and were also visible in the patterns of the composites. The diffraction peaks at $25.8^{\circ}, 31.7^{\circ}, 32.9^{\circ}, 39.7^{\circ}, 46.7^{\circ}$, and $49.6^{\circ}$ in $2 \theta$ revealed the presence of calcium phosphate (Figures 4(b), 4(c), and 4(d)). Moreover, the intensity of the peaks in the X-ray diffraction pattern of carboxymethylated cellulose precalcified with calcium hydroxide (Figure 4(d)) was higher in comparison to that of other samples (Figures 4(b) and 4(c)) due to bigger crystals on the surface. This was also visible from SEM micrographs (Figure 5). Large aggregates appeared on the surface of the carboxymethylated cellulose matrix preactivated with calcium hydroxide after exposure to 1.5x SBF (Figure 5). However, small hydroxyapatite crystals covered both the cellulose pretreated with calcium and phosphate ions solutions and the cellulose matrix pretreated with the calcium silicate solution.

As well as precalcification methods, the results obtained from studies on biomimetic mineralization underlined how the type of functional groups on the cellulose surface played an important role not only for the variation in the size of the hydroxyapatite crystals but also for the deposited mass onto the surface (Table 1) like it was observed by other researchers $[21,23,25,26]$.

Our results also showed that the precalcification methods and the type of functional groups influenced the mineralization rate. As can be seen from the results, the highest mineralization rate was achieved on the carboxymethylated cellulose

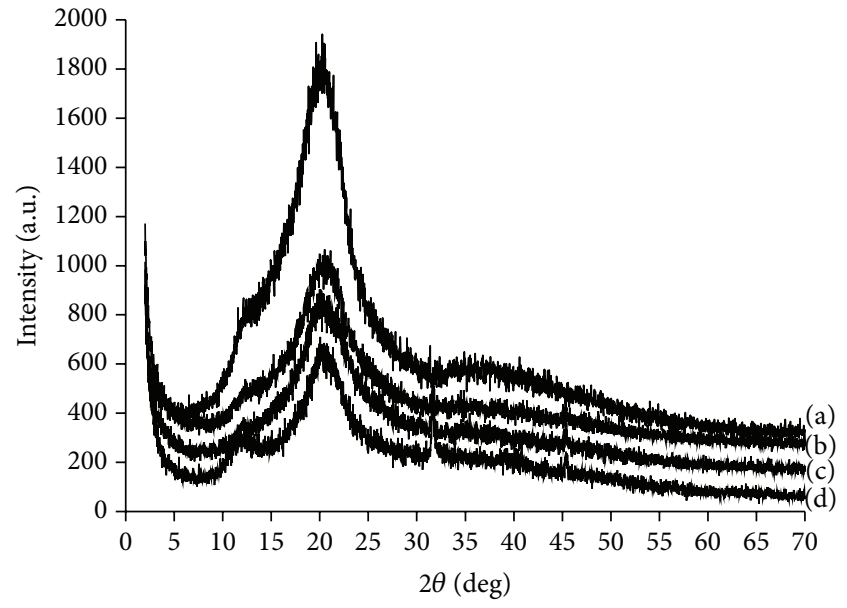

FIGURE 4: X-ray diffraction patterns of (a) regenerated cellulose; (b) mineralized cellulose pretreated with the calcium silicate solution; (c) mineralized cellulose pretreated with calcium and phosphate ions solutions; (d) mineralized carboxymethylcellulose preactivated with a saturated $\mathrm{Ca}(\mathrm{OH})_{2}$ solution.

matrix, which was activated with the calcium hydroxide solution. Also the mass percentage of the hydroxyapatite fraction was two times larger after a few days. We assume that the higher rate of mineralization is required for the faster alteration of cellulose surface chemistry leading to an enhanced bioactivity and biocompatibility.

Matching the nanostructure of the inorganic phase of natural bone, most studies are focused on the development of scaffolds with nanohydroxyapatite. Shi et al. [27] investigated the influence of the size of hydroxyapatite on proliferation and apoptosis of osteoblast-like cells. The results showed that nanohydroxyapatite can be a better candidate in biomedical applications than microhydroxyapatite. However, we assume that nanohydroxyapatite crystals can grow and form the morphology of cauliflower in microscale (Figure 5(d)). Our findings agree with the results of Beşkardeş and Gumusderelioglu [28].

After 9 weeks of incubation, further observations showed that the matrices started to degrade (data are not presented). For these studies, it is very important that the matrix is biodegradable, as new bone would have to replace it. The degradation of the material and the whole replacing with bone tissue cell are the main objective of the current research on grafting materials. Numerous biomaterials used like scaffolds have been available for the bone regenerative 


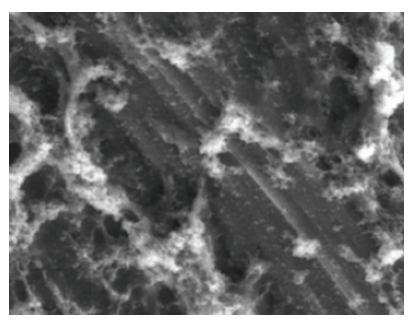

(a)

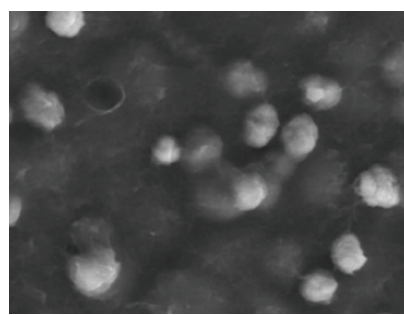

(d)

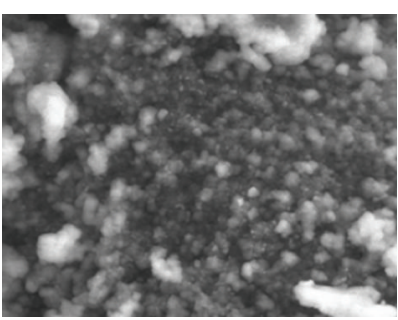

(b)

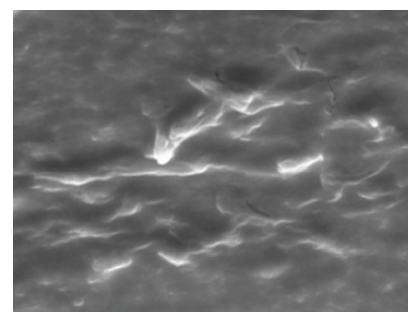

(e)

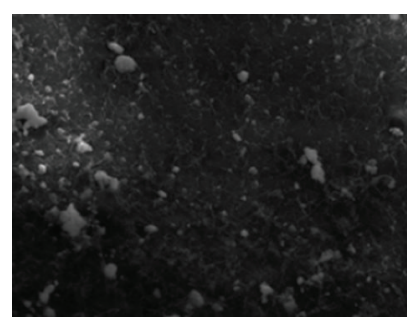

(c)

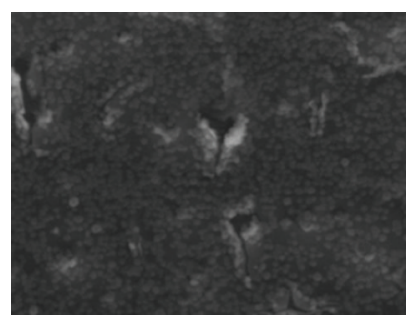

(f)

FIGURE 5: SEM micrographs of the surface of (a) the cellulose matrix treated with calcium and phosphate ions solutions then (b) soaked in $1.5 \mathrm{x}$ SBF; (c) the matrix activated with $\mathrm{Ca}(\mathrm{OH})_{2}$ then $(\mathrm{d})$ soaked in $1.5 \mathrm{x}$ SBF; (e) the matrix treated with the calcium silicate solution and then (f) soaked in $1.5 \mathrm{x}$ SBF.

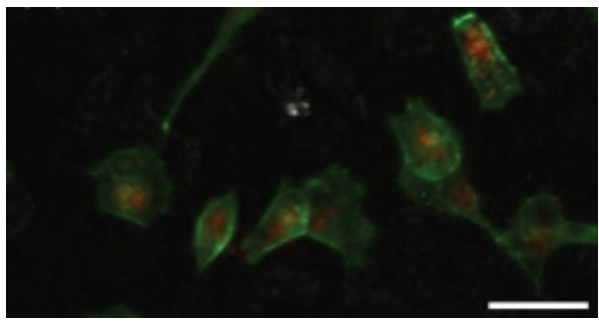

(a)

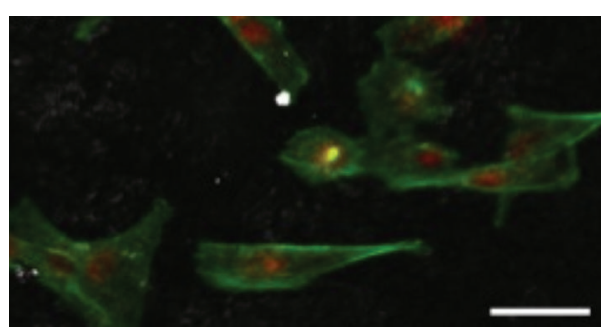

(c)

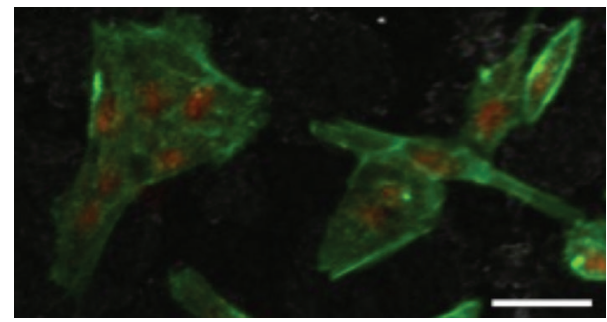

(b)

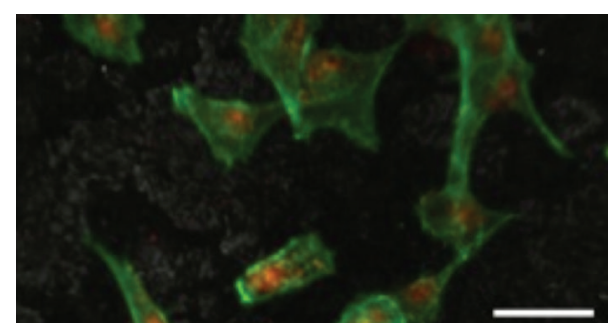

(d)

FIGURE 6: CLSM images of MG63 osteoblastic cells cultured for 24 hours over (a) cellulose matrix, (b) mineralized cellulose pre-treated with calcium and phosphate ions solutions, (c) mineralized carboxymethylated cellulose pre-treated with a saturated $\mathrm{Ca}(\mathrm{OH})_{2}$ solution, and $(\mathrm{d})$ mineralized cellulose pre-treated with the calcium silicate solution. Scale bar corresponds to $40 \mu \mathrm{m}$.

techniques, unfortunately, most of these medical devices are expensive and still present unpredictable biological results $[9,16,21,24,29,30]$.

3.3. Biological Evaluation of the Mineralized Cellulose Substrates. The biological characterization of the cellulose matrix (control scaffold) and the three developed mineralized cellulose samples was conducted with MG63 human osteoblastic cells, for a period of 7 days.
Observation of the material samples by CLSM at 1 day of culture showed well spread cells with an elongated/polygonal morphology, and establishing cell-to-cell contact, both on the cellulose matrix (control) and on the mineralized cellulose samples (Figure 6). On the assayed mineralized cellulose samples, adhered cells were found to be more expanded, with an increased cytoplasmic volume and higher number of fibrillar projections, as comparing to control. In addition, in all experimental conditions, cells exhibited a well-organized 


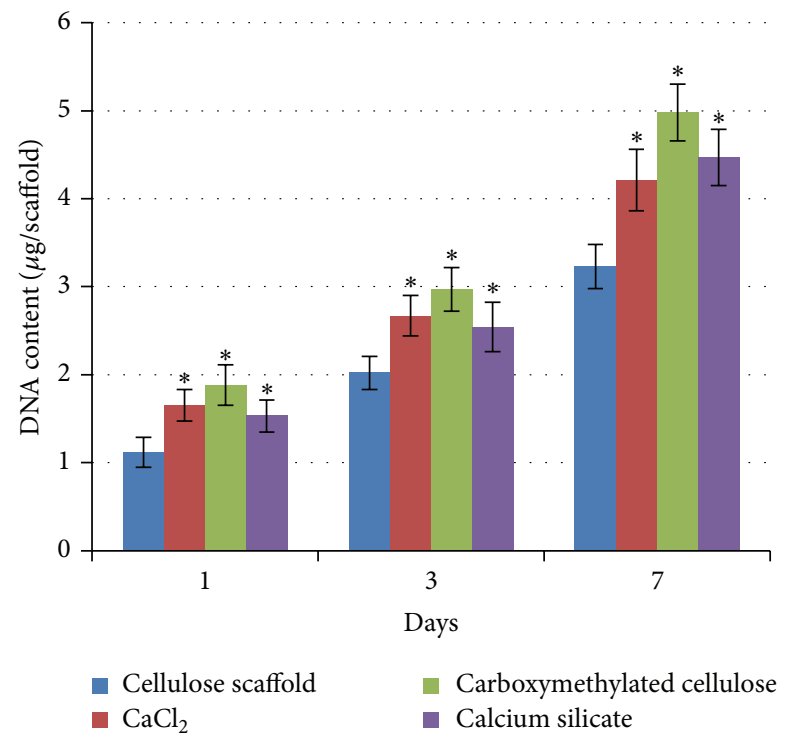

FIGURE 7: DNA content of MG63 osteoblastic cell cultures grown over the cellulose matrix and the mineralized cellulose matrices. ${ }^{*}$ Significantly different from the cellulose matrix.

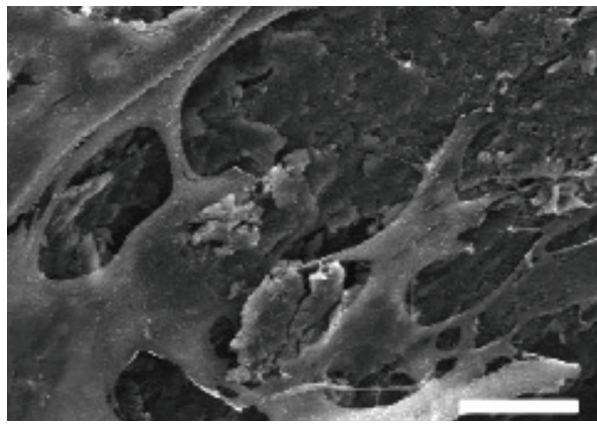

(a)

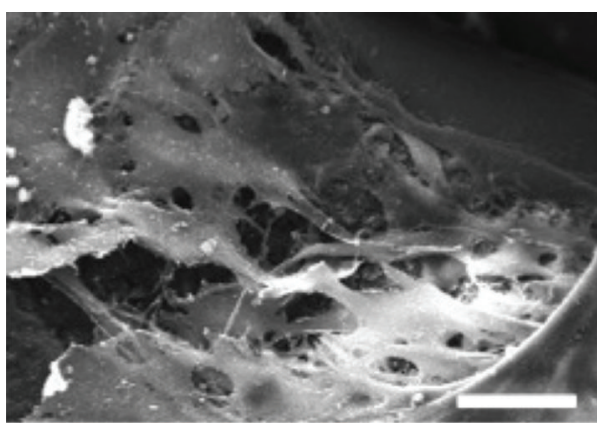

(c)

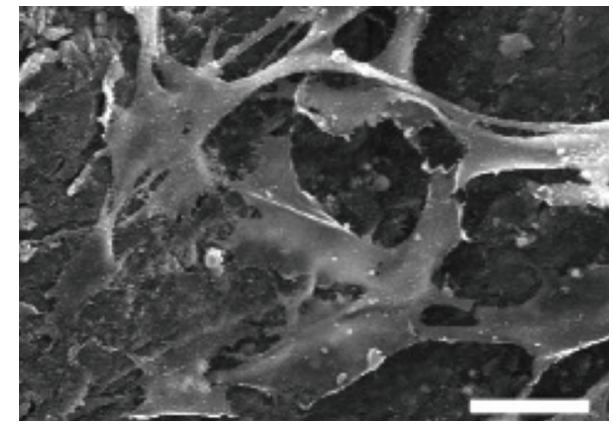

(b)

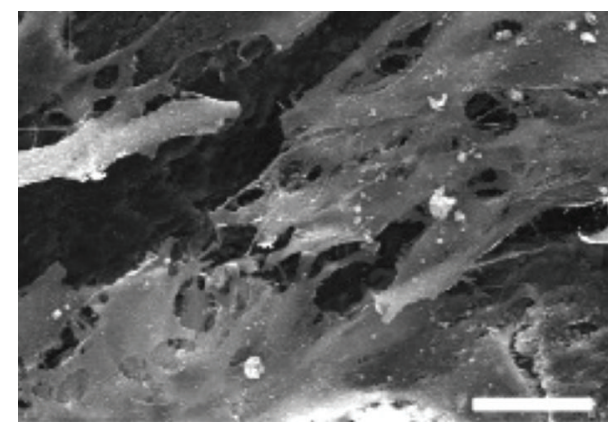

(d)

FIGURE 8: SEM images of MG63 osteoblastic cells cultured for 3 days over (a) cellulose matrix, (b) mineralized cellulose pre-treated with calcium and phosphate ions solutions, (c) mineralized carboxymethylated cellulose pre-treated with a saturated $\mathrm{Ca}(\mathrm{OH})_{2}$ solution, and $(\mathrm{d})$ mineralized cellulose pre-treated with the calcium silicate solution. Scale bar corresponds to $30 \mu \mathrm{m}$.

F-actin cytoskeleton, with intense staining at the cell boundaries, prominent nucleus and on-going cell division, which are signs of a healthy cellular behaviour [31]. This information is relevant in terms of the biological performance of the scaffolds, as the F-actin cytoskeleton, which is highly concentrated just beneath the plasma membrane, provides structural stability and elasticity to the cell undergoing adaptation to the substrate topography [31]. Also, the F-actin cytoskeleton is a key player in the cellular mechanotransduction mechanisms modulating complex signalling pathways essential to the subsequent stages of osteoblastic proliferation and differentiation $[26,31-33]$. 
Assessment of DNA content showed that the cellulose matrix supports the proliferation of the osteoblastic cells, with increasing values throughout the 7-day culture period. Comparatively, it is worth to note the significantly increased values observed at day 1 for the mineralized cellulose matrices, suggesting a higher number of attached cells over these substrates. Furthermore, DNA content at days 3 and 7 was also higher in the mineralized cellulose scaffolds. Cultures grown on carboxymethylated cellulose presented, throughout the culture time, the highest values for total DNA content. However, differences among the three mineralized samples were not statistically significant. Results are summarized in Figure 7.

SEM imaging revealed that cells adhered well to the control and the mineralized cellulose matrices attaining a spreading pattern and showing a perfect adaptation to the underlying topography. Representative images are shown for cultures with 3 days (Figure 8), and the presence of an organized cell layer covering partially the material surface was evident.

The observed results ensure that the developed cellulose porous matrix is not cytotoxic and can be used in a contact with biological systems. In addition, the presence of a hydroxyapatite layer in the originally prepared cellulose matrix clearly improved cell adhesion, as shown by the higher DNA content at day 1 found in the three mineralized matrices. Also, a higher cell growth rate was evident throughout the culture time, compared to the control cellulose matrix. These results are in line with a variety of studies suggesting that cellulose is a useful scaffolding material in regenerative medicine, including in bone tissue applications, reporting the adhesion, proliferation, and differentiation of osteoblastic lineage cells $[19,34-36]$. Further, there is evidence that the presence of a calcium phosphate containing layer on the surface of cellulose substrates promotes cell adhesion and growth, due to the biomimetic nature of the resulting surface $[2,19,37,38]$.

\section{Conclusions}

A highly porous cellulose matrix was successfully created by lyophilisation of a cellulose gel. The behaviour of MG-63 human osteoblastic cells on the investigated cellulose matrix confirmed it was not cytotoxic as cells were able to adhere and proliferate on it. It was found that the cellulose matrix containing silanol, carboxyl, or/and hydroxyl groups combined with calcium ions induced hydroxyapatite formation on its surface during the biomimetic mineralization. A fully covered surface was obtained during two weeks of storage in simulated body fluid at a temperature of $37^{\circ} \mathrm{C}$.

Comparison of different matrix precalcification methods showed that the highest mineralization rate and larger deposited mass of hydroxyapatite were achieved on the matrix of carboxymethylated cellulose activated with a calcium hydroxide solution. The porous cellulose matrix was not cytotoxic, allowing the adhesion and proliferation of human osteoblastic cells. Comparatively, improved cell adhesion and growth rate were achieved on the mineralized cellulose matrices.

\section{Conflict of Interests}

all the authors have no conflict of interests regarding the devices used for this study, and the current research is not influenced by any secondary interest, such as financial gain.

\section{Acknowledgments}

The studies were performed in a frame of COST CM1101 project Colloidal Aspects of Nanoscience for Innovative Processes and Materials. The authors want to thank Veronica Gavin B. Sc., T.E.F.L, for English grammar proof on the paper.

\section{References}

[1] H. Li, C. R. Zhou, M. Y. Zhu, J. H. Tian, and J. H. Rong, "Preparation and characterization of homogeneous hydroxyapatite/chitosan composite scaffolds via in-situ hydration," Journal of Biomaterial and Nanobiotechnology, vol. 1, pp. 42-49, 2010.

[2] K. A. Zimmermann, J. M. Leblanc, K. T. Sheets, R. W. Fox, and P. Gatenholm, "Biomimetic design of a bacterial cellulose/hydroxyapatite nanocomposite for bone healing applications," Materials Science and Engineering C, vol. 31, no. 1, pp. 43-49, 2011.

[3] A. S. Herford, R. Tandon, T. W. Stevens, E. Stoffella, and M. Cicciù, "Immediate distraction osteogenesis: the sandwich technique in combination with rhBMP-2 for anterior maxillary and mandibular defects," Journal of Craniofacial Surgery, vol. 24, no. 4, pp. 1383-1387, 2013.

[4] A. R. Boccaccini and V. Maquet, "Bioresorbable and bioactive polymer/Bioglass composites with tailored pore structure for tissue engineering applications," Composites Science and Technology, vol. 63, no. 16, pp. 2417-2429, 2003.

[5] C. Y. Zhang, H. Lu, Z. Zhuang, X. P. Wang, and Q. F. Fang, "Nano-hydroxyapatite/poly(L-lactic acid) composite synthesized by a modified in situ precipitation: preparation and properties," Journal of Materials Science, vol. 21, no. 12, pp. 30773083, 2010.

[6] A. S. Herford, M. Lu, L. Akin, and M. Cicciù, "Evaluation of a porcine matrix with and without platelet-derived growth factor for bone graft coverage in pigs," International Journal of Oral and Maxillofacial Implants, vol. 27, no. 6, pp. 1351-1358, 2012.

[7] O. Petrauskaite, J. Liesiene, C. Santos et al., "Nano-hydroxyapatite/cellulose composite scaffold for bone tissue engineering," Journal of Tissue Engineering and Regenerative Medicine, vol. 6, p. 34, 2012.

[8] M. I. Sabir, X. Xu, and L. Li, "A review on biodegradable polymeric materials for bone tissue engineering applications," Journal of Materials Science, vol. 44, no. 21, pp. 5713-5724, 2009.

[9] M. Jarcho, J. F. Kay, K. I. Gumaer, R. H. Doremus, and H. P. Drobeck, "Tissue, cellular and subcellular events at a boneceramic hydroxylapatite interface," Journal of Bioengineering, vol. 1, no. 2, pp. 79-92, 1977.

[10] G. Chen, T. Ushida, and T. Tateishi, "Scaffold design for tissue engineering," Macromolecular Bioscience, vol. 2, pp. 67-77, 2002.

[11] D. W. Hutmacher, T. Schantz, I. Zien, K. W. Ng, K. H. Teoh, and K. C. Tan, "Mechanical properties and cell cultural response of polycaprolactone scaffolds designed and fabricated via fused deposition modeling," Journal of Biomedical Material Research, vol. 55, pp. 203-216, 2001. 
[12] L. S. Nair and C. T. Laurencin, "Polymers as biomaterials for tissue engineering and controlled drug delivery," Advances in Biochemical Engineering/Biotechnology, vol. 102, pp. 47-90, 2006.

[13] M. V. Risbud and R. R. Bhonde, "Suitability of cellulose molecular dialysis membrane for bioartificial pancreas: in vitro biocompatibility studies," Journal of Biomedical Material Research, vol. 54, pp. 436-444, 2001.

[14] M. Märtson, J. Viljanto, T. Hurme, and P. Saukko, "Biocompatibility of cellulose sponge with bone," European Surgical Research, vol. 30, no. 6, pp. 426-432, 1998.

[15] Y. S. Nam, J. J. Yoon, and T. G. Park, "A novel fabrication method of macroporous biodegradable polymer scaffolds using gas forming salt as a porogen additive," Journal Biomedical Material Research, vol. 53, pp. 1-7, 2000.

[16] M. Kawashita, M. Nakao, M. Minoda et al., "Apatite-forming ability of carboxyl group-containing polymer gels in a simulated body fluid," Biomaterials, vol. 24, no. 14, pp. 2477-2484, 2003.

[17] M. Tanahashi and T. Matsuda, "Surface functional group dependence on apatite formation on self-assembled monolayers in a simulated body fluid," Journal Biomedical Material Research, vol. 34, pp. 305-315, 1997.

[18] M. Cicciù, A. S. Herford, E. Stoffella, G. Cervino, and D. Cicciù, "Protein-signaled guided bone regeneration using titanium mesh and Rh-BMP2 in oral surgery: a case report involving left mandibular reconstruction after tumor resection," Open Dentistry Journal, vol. 6, no. 1, pp. 51-55, 2012.

[19] L. Hong, Y. L. Wang, S. R. Jia, Y. Huang, C. Gao, and Y. Z. Wan, "Hydroxyapatite/bacterial cellulose composites synthesized via a biomimetic route," Materials Letters, vol. 60, no. 13-14, pp. 1710-1713, 2006.

[20] T. T. Nge and J. Sugiyama, "Surface functional group dependent apatite formation on bacterial cellulose microfibrils network in a simulated body fluid," Journal of Biomedical Materials Research A, vol. 81, no. 1, pp. 124-134, 2007.

[21] I. B. Leonor, E. T. Baran, M. Kawashita, R. L. Reis, T. Kokubo, and T. Nakamura, "Growth of a bonelike apatite on chitosan microparticles after a calcium silicate treatment," Acta Biomaterialia, vol. 4, no. 5, pp. 1349-1359, 2008.

[22] J. Bryjak, J. Aniulyte, and J. Liesiene, "Evaluation of mantailored cellulose-based carriers in glucoamylase immobilization," Carbohydrate Research, vol. 342, no. 8, pp. 1105-1109, 2007.

[23] Y. Zhang and M. Zhang, "Synthesis and characterization of macroporous chitosan/calcium phosphate composite scaffolds for tissue engineering," Journal Biomedical Material Research, vol. 55, pp. 304-312, 2001.

[24] Ž. P. Stojanović, K. Jeremić, S. Jovanović, W. Nierling, and M. D. Lechner, "Light scattering and viscosity investigation of dilute aqueous solutions of carboxymethyl starch," Starch, vol. 61, no. 3-4, pp. 199-205, 2009.

[25] P. Cromme, C. Zollfrank, L. Müller, F. A. Müller, and P. Greil, "Biomimetic mineralisation of apatites on $\mathrm{Ca}^{2+}$ activated cellulose templates," Materials Science and Engineering $C$, vol. 27, no. 1, pp. 1-7, 2007.

[26] A. I. Rodrigues, M. E. Gomes, I. B. Leonor, and R. L. Reis, "A biological scaffold filled with silica and simultaneously crosslinked with polyurethane," Acta Biomaterials, vol. 8, pp. 3765-3776, 2012.

[27] Z. Shi, X. Huang, Y. Cai, R. Tang, and D. Yang, "Size effect of hydroxyapatite nanoparticles on proliferation and apoptosis of osteoblast-like cells," Acta Biomaterialia, vol. 5, no. 1, pp. 338345, 2009.
[28] I. G. Beşkardeş and M. Gumusderelioglu, "Biomimetic apatitecoated PCL scaffolds: effect of surface nanotopography on cellular functions," Journal of Bioactive and Compatible Polymers, vol. 24, no. 6, pp. 507-524, 2009.

[29] M. J. Olszta, X. Cheng, S. S. Jee et al., "Bone structure and formation: a new perspective," Materials Science and Engineering $R$, vol. 58, no. 3-5, pp. 77-116, 2007.

[30] A. Yokoyama, M. Gelinsky, T. Kawasaki et al., "Biomimetic porous scaffolds with high elasticity made from mineralized collagen-an animal study," Journal of Biomedical Materials Research B, vol. 75, no. 2, pp. 464-472, 2005.

[31] B. Alberts, A. Johnson, J. Lewis, M. Raff, K. Roberts, and P. Walter, "The cytoskeleton," in Molecular Biology of the Cell, B. Alberts, A. Johnson, J. Lewis, M. Raff, K. Roberts, and P. Walter, Eds., pp. 907-982, Garland, New York, NY, USA, 4th edition, 2002.

[32] D. Stamenovíc, "Effects of cytoskeletal prestress on cell rheological behavior," Acta Biomaterialia, vol. 1, no. 3, pp. 255-262, 2005.

[33] I. Titushkin and M. Cho, "Modulation of cellular mechanics during osteogenic differentiation of human mesenchymal stem cells," Biophysical Journal, vol. 93, no. 10, pp. 3693-3702, 2007.

[34] Y. M. Chen, T. Xi, Y. Zheng et al., "In vitro cytotoxicity of bacterial cellulose scaffolds used for tissue-engineered bone," Journal of Bioactive and Compatible Polymers, vol. 24, no. 1, pp. 137-145, 2009.

[35] S. Saska, H. S. Barud, A. M. M. Gaspar, R. Marchetto, S. J. L. Ribeiro, and Y. Messaddeq, "Bacterial cellulose-hydroxyapatite nanocomposites for bone regeneration," International Journal of Biomaterials, vol. 2011, Article ID 175362, 8 pages, 2011.

[36] A. Aravamudhan, D. M. Ramos, J. Nip et al., "Cellulose and collagen derived micro-nano structured scaffolds for bone tissue engineering," Journal Biomedical Nanotechnology, vol. 9, pp. 719-731, 2013.

[37] B. Fang, Y.-Z. Wan, T.-T. Tang, C. Gao, and K.-R. Dai, "Proliferation and osteoblastic differentiation of human bone marrow stromal cells on hydroxyapatite/bacterial cellulose nanocomposite scaffolds," Tissue Engineering A, vol. 15, no. 5, pp. 10911098, 2009.

[38] C. J. Grande, F. G. Torres, C. M. Gomez, and M. Carmen Bañó, "Nanocomposites of bacterial cellulose/hydroxyapatite for biomedical applications," Acta Biomaterialia, vol. 5, no. 5, pp. 1605-1615, 2009. 

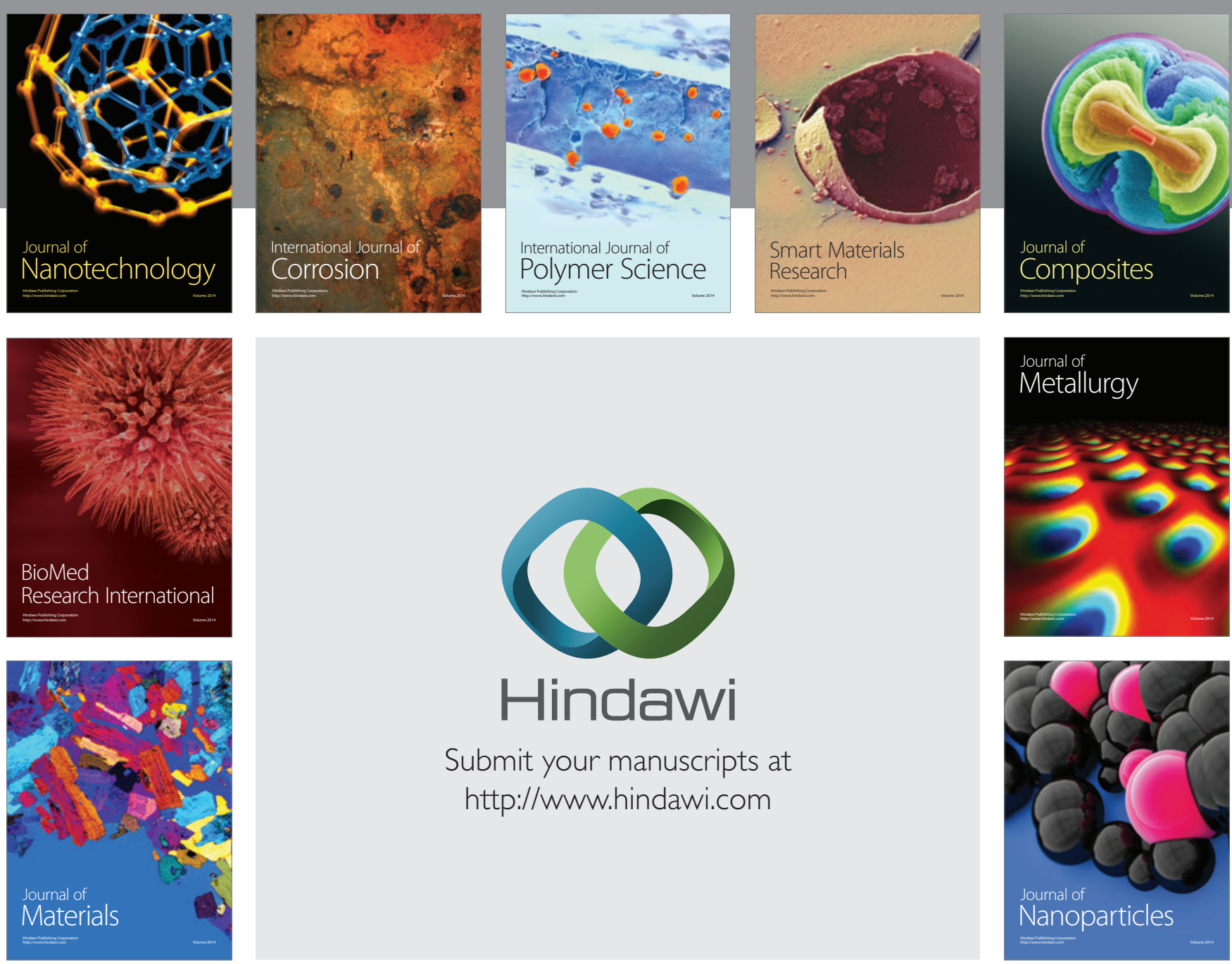

Submit your manuscripts at http://www.hindawi.com
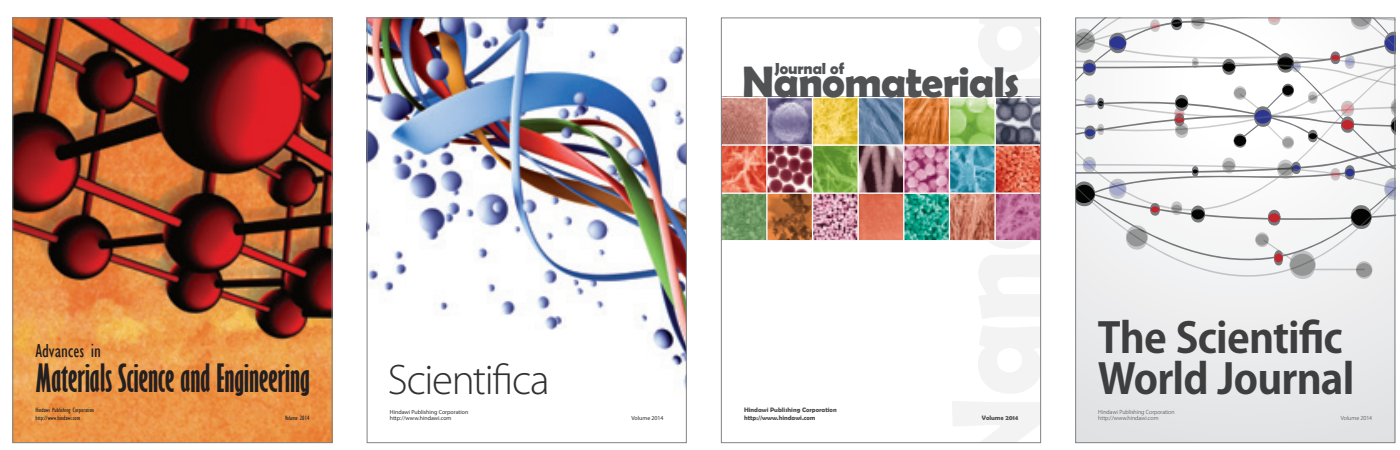

\section{The Scientific World Journal}
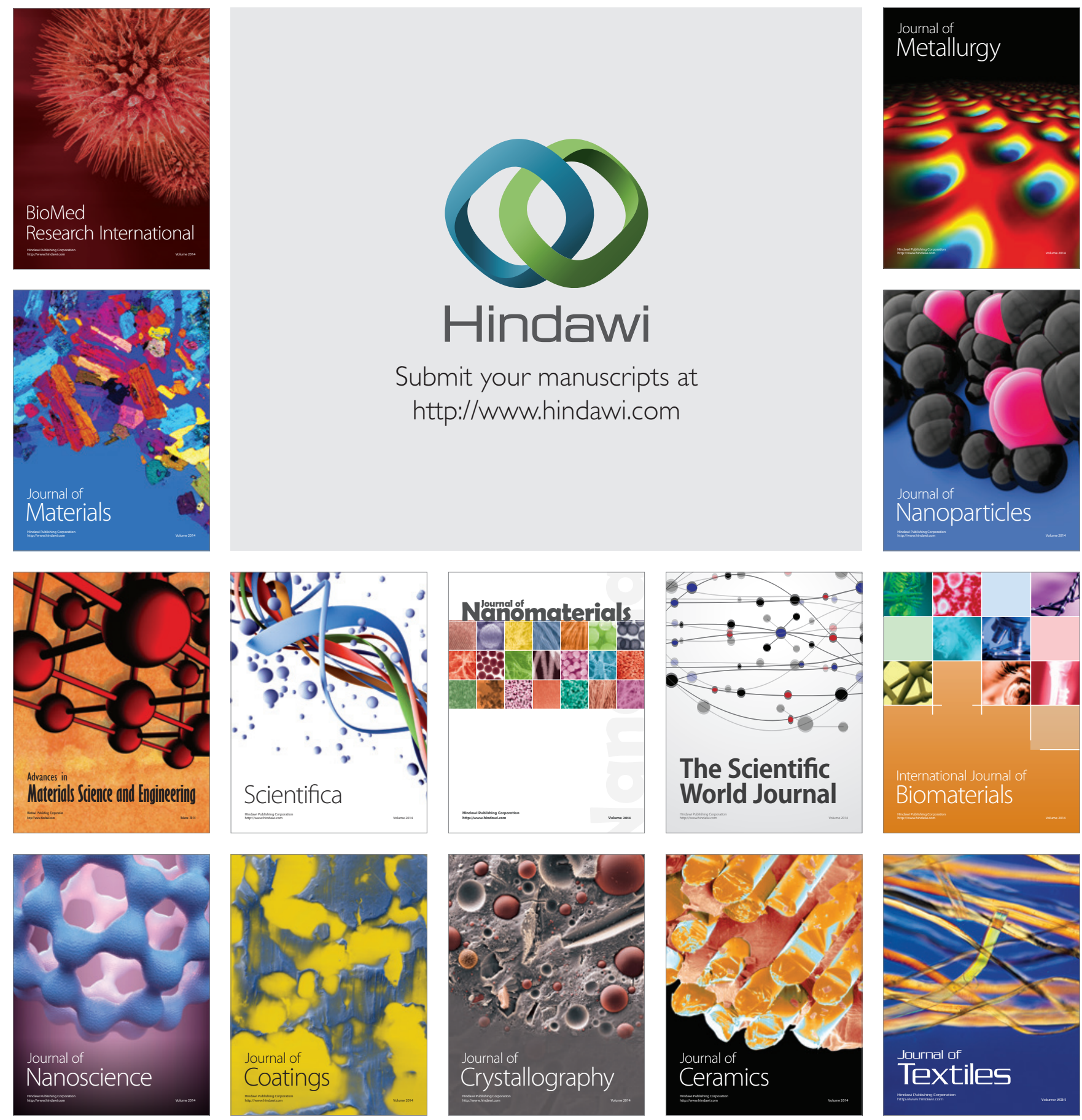